\title{
Fast Remote data access for control of TCP/IP network using android Mobile device
}

\author{
Vaibhav Muddebihalkar ${ }^{1}$, R.M. Goudar ${ }^{2}$ \\ I'(Department, of Computer Engineering MIT AOE Alandi, University of Pune,India,) \\ ${ }^{2}$ (Department, of Computer Engineering MIT AOE Alandi, University of Pune,India,)
}

\begin{abstract}
In today's world most of the mobile have the use more than its basic functionality. As mobile becomes more advance to be have same architecture same as desktop system. Hence this feature should be used as to create application that controls the desktop nodes in the network with help of client server architecture. This paper purpose $x$ The application connects the system node by server and server connects to android mobile which will easy to control network from any mobile place. The application having functionality to capture a screenshot of client node to know the state of system which will have to capture picture and send over network which will required some time. By adding compression technique for image as screenshot which helps to send faster over the network. This all implemented using JAVA and servlets which also increase performance
\end{abstract}

Keywords: Android, Remote control, JAVA-Servlets, image compression.

\section{Introduction}

Smart-phones nowadays mostly build with configuration which leads to have more features. Theses mobile can be used to execute task that same as performed on the desktop system. Remote control system can be used to by desktop system for controlling network of system nodes, but these systems required a wired connection between them. [2] So whenever if administrator is outside of campus then it is not possible to contact to network or control it. Smart-phones are having good network connectivity options like 3G, Wi-Fi which helps to perform task easier and faster on mobile devices. Previous protocols like WAP are now replaced by direction connection protocol for the network. These changes not restrict the remote control system to limited feature.[1]

To create the remote control for the system architecture performs functions which uses software stacks to analyze current state of system. To connect to systems communication standards should be used to create a stable communication. Wireless communication should be standard platform but it is not a safe communication, so wired connection also preferred.[1]

Architecture should be stable but requires some security as data is transferred from client to server. So security algorithm is implemented for file transfer as commands are not much important.[1]

The architecture purposes the functionality which captures screenshots of the client system and sends to server and from server to smart-phone. This procedure requires the lot a time as sending image require lot of transfers of data and also it dependent network bandwidth. The compression technique allows compressing file and sending over network which will reduce the time, helpful to improve performance of architecture. [3][4].

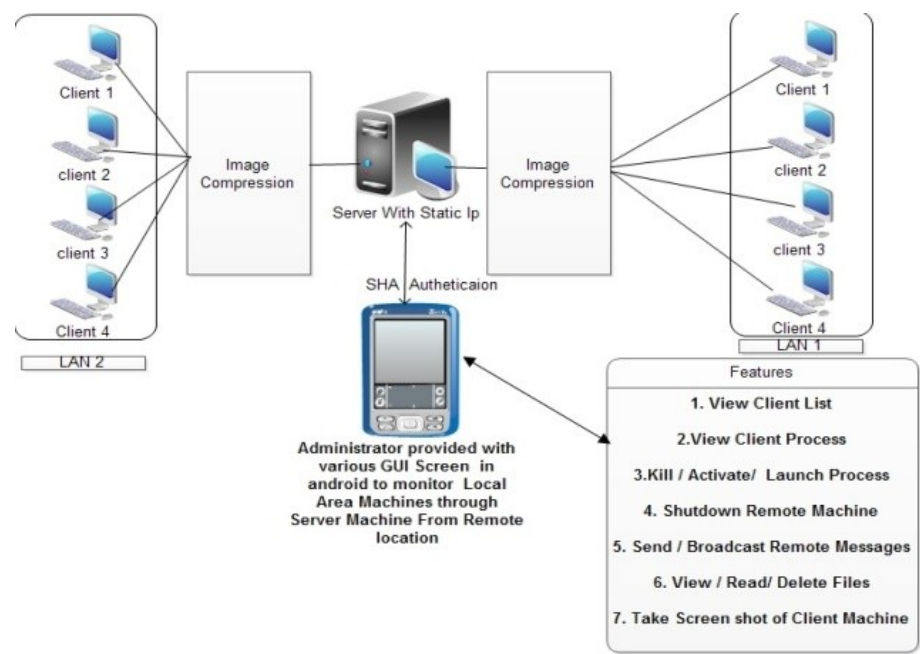

Fig 1.System Architecture 


\section{The Architecture (11 Bold)}

The architecture is consisting of the major 3 modules which will be consisting server module, client module and mobile application. In this architecture also consist of the image compression algorithm which will include in the client part. The flow of architecture includes running client demon on the numbers of node in the networks. After that sever module will be started, which immediately collecting all information of client nodes, which is connected to server.[3][7] So with help of the server graphical interface number of operation will be executed like controlling process, file operations, sending message capturing screenshots. These all operations should handle by mobile application which fetch information server. Mobile should be connected through either internet connection or Wi-Fi connection. To perform operation from server module user have to first select particular client from list of clients. The operations which are performed from server module are controlling processes which start new process and kill running process on client node. For initialization of new process required to type name of process. To kill running process user have to select process from list of processes. File operation consist of the list of files from a particular path. By using particular path user can create and delete file from that folder. To read particular file user have to enter path of file and can accesses file. To send the message to a particular system user have enter message and press send button and if user wants to send same message to all clients then it will be done by send-to-all button. The screenshot operation should be applicable after selecting particular client and pressing screenshot button.

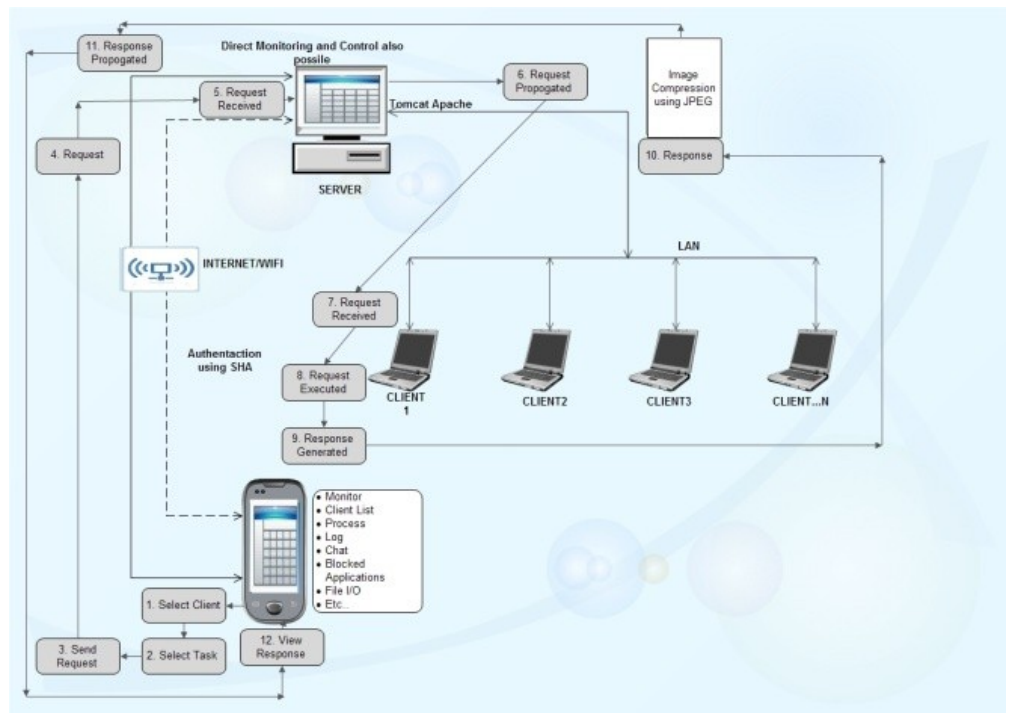

Fig 2 System Process Flow

\section{A Server Module:-}

The server module should be consisting of the graphical user interface which includes the all specific operations. Server module is coded with help of Java and servlets. Server module designed with help of JAVA Swing application framework. The connectivity assignment should be done with URL class of java which connects the client to server with a particular url of server which also include IP address of server. This same URL package can be used for sending and receiving data from client and server. To exchange data java object serialization was used. The servlets coding was used to retrieve data from client node and also used to send data. Server application continuously checks for new entry of client in to list and accepting data from client. Server application should perform operation which is listed in architecture as control of process, files operation, sending messages and capturing screenshot. Server application fetch files form client node of only text formats due size limitation. To remove or to stop any node from network shutdown operation was performed. As all commands and files are transferred over network, security should be provide with adding AES algorithm was included. 


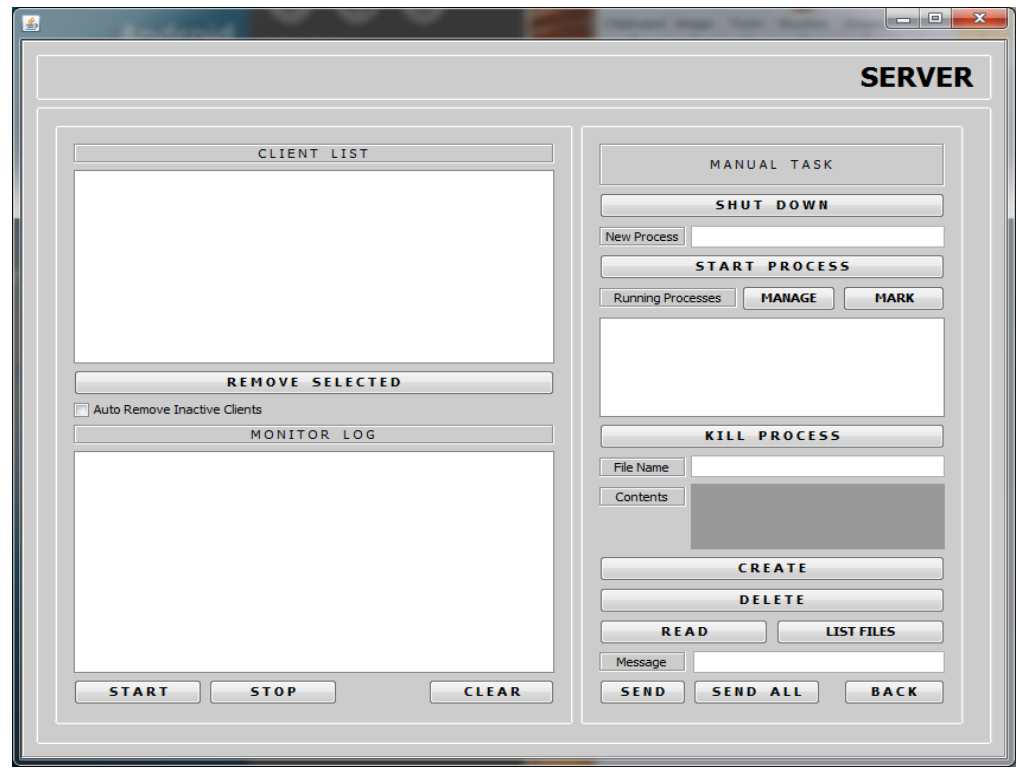

Fig3. Server GUI.

\section{B Client Module:-}

The client module was installed on the each client node which is connected in network. Client module has GUI which helps user to connect to server. The graphical interface consists of one field to enter IP address of the server and one button to enable or disable client in application network. The client GUI also has one display area which display all information regarding which commands was executed by server on that client node. This display area also put messages which are sent by server. The client module also uses two class of JAVA as process and process builder to manipulate processes which running on client node. The java object serialization was used to send and receive object and convert them into byte which helps to easy transfer over network.

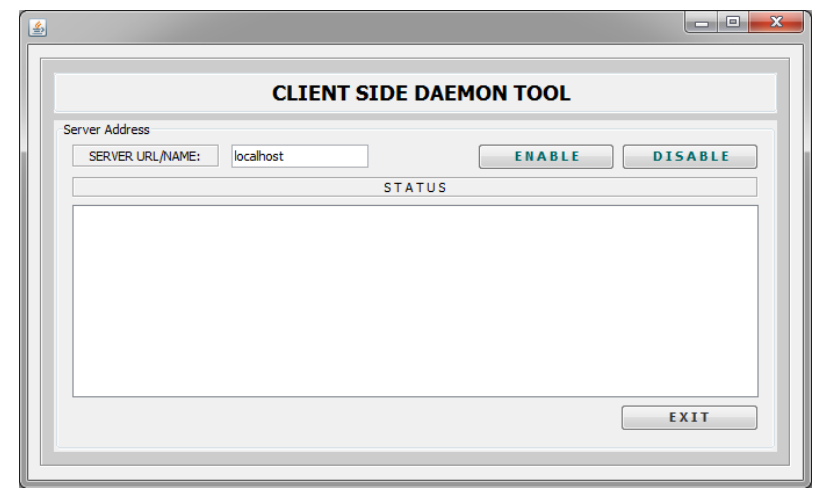

Fig4 Client GUI

\section{Android Client:-}

This is mobile application module which runs on the smart-phone. This application has same functionality as server module. The smart-phone on which this module will run was connected to server with internet connectivity or Wi-Fi connectivity. If smart-phone is connected to server with internet connectivity then server will be provided with static IP address. The mobile application start with asking IP address of server to connect and password. After successful login next screen will display list of client which was connected to server and by selecting one client at a time user will be provide with same functions as server module. 


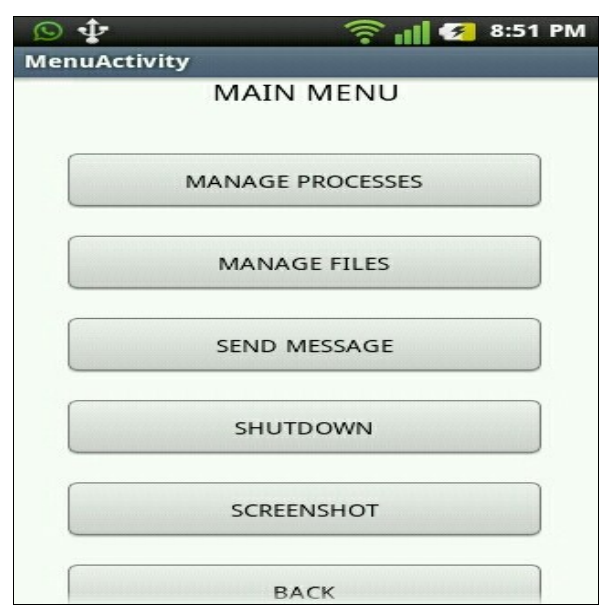

Fig5 Android GUI for controlling client.

\section{Implementation}

The implementation of application has some procedure which is as follows.

When all clients get connected to the server then user can control the one client at a time with help of operation described in architecture. Android smart-phone also connected to server which will fetch all information from server. To operate from android client we need some procedure to be follow.

1) Enter IP address and passkey to make login into system.

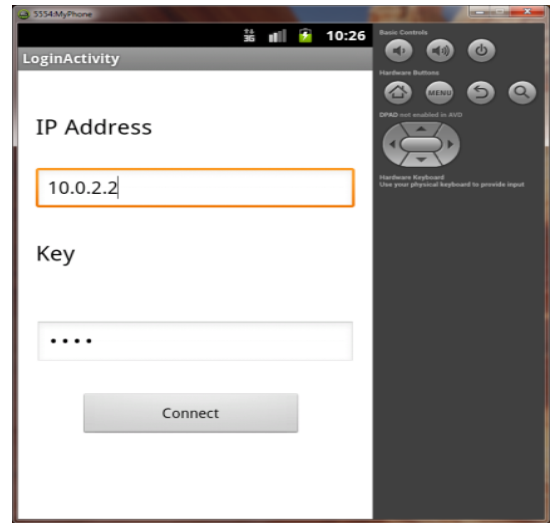

Fig6. To make login to system using android Phone

2) After making successful login user can see the list of client connected to server and can select one client to proceed for operation.

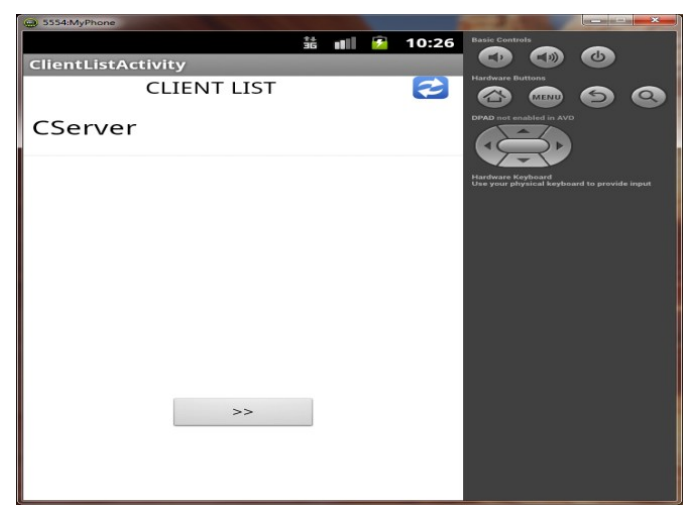

Fig7. List of clients.

2) At last user can see the list of operation to be executed on client. Which is already given in fig 5 . 


\section{Image compression}

As for getting screenshot of system user will be aware of current state of the system. So after capturing screenshot at client node with help of the client module, this image was sent over network to server and then sent it to mobile application. To perform this procedure it requires lot of bytes transferred over network, hence it requires a lot of time. To reduce time and improve performance of system one solution is to first compress the image and then transfer over the network. By compressing image it size will be get reduced and it was easy and faster to transfer over the network. To do image compression lot of lossless algorithms was purposed which reduces size of image without losing and information. So we first tried with Huffman coding algorithm for image compression, but it took lot of time to compress image on client side and then it will send on network. so algorithm which take less time to compress image was JPEG compression. So we used JPEG algorithm to compress image which take minimum time. [6]

\begin{tabular}{|c|c|c|}
\hline \multirow{2}{*}{ Algorithm } & \multicolumn{2}{|c|}{ CPU Time } \\
\cline { 2 - 3 } & Encoding & Decoding \\
\hline Wavelet & $0.35 \mathrm{Sec}$ & $0.27 \mathrm{Sec}$ \\
\hline JPEG & $0.12 \mathrm{Sec}$ & $0.12 \mathrm{Sec}$ \\
\hline VQ & $2.45 \mathrm{Sec}$ & $0.18 \mathrm{Sec}$ \\
\hline Fractal & $5.65 \mathrm{hrs}$ & $1.35 \mathrm{Sec}$ \\
\hline
\end{tabular}

Table 1Comparision of performance of coding algorithms on $256 \times 256$ images [4]

\begin{tabular}{|c|c|c|}
\hline Algorithm & Encoding Time & Decoding Time \\
\hline Wavelet & $0.8 \mathrm{Sec}$ & $0.7 \mathrm{Sec}$ \\
\hline JPEG & $0.2 \mathrm{Sec}$ & $0.2 \mathrm{Sec}$ \\
\hline VQ & $6.0 \mathrm{Sec}$ & $0.7 \mathrm{Sec}$ \\
\hline Fractal & $6.3 \mathrm{hrs}$ & $3.5 \mathrm{Sec}$ \\
\hline
\end{tabular}

Table 2 Comparison of Performance of Coding Algorithms on 400×400 Images [4].

\begin{tabular}{|c|c|}
\hline Method & Compression Ratio \\
\hline Wavelet & $>>32$ \\
\hline JPEG & $<=50$ \\
\hline VQ & $<32$ \\
\hline Fractal & $>=16$ \\
\hline
\end{tabular}

Table 3 Comparison of Performance On The Basis Of Compression Ratio of Different Coding Algorithms.

By this comparison we can conclude that JPEG takes less CPU time to perform encoding and decoding of the image compression. As well as JPEG also high compression ration compared to remaining image compression algorithms.

\section{Results}

By using JPEG compression algorithm for image compression image it is helps to transfer image faster over network. As JPEG is lossy technique but it after receiving image on the Android mobile application it good to know details. We mostly used time parameter to compare existing and current system. We tested application as on different client machine which have different hardware configuration and different windows operation system. So results are very good as current system takes very less time to capture and send screen shot from client to server.

\begin{tabular}{|l|l|l|}
\hline \multirow{2}{*}{ Operation } & \multicolumn{2}{|l|}{ Time Required (Seconds) } \\
\cline { 2 - 3 } & windows 8 & windows xp \\
\hline Capture Screenshot & 8 & 9 \\
\hline
\end{tabular}

Table 4 Time required taking and sending screenshot without compression.

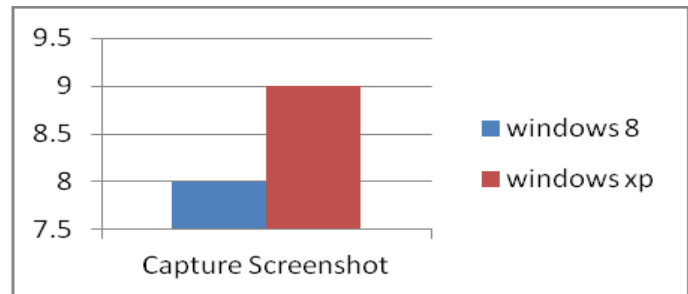

Fig 9 More time required for system without using compression. 


\begin{tabular}{|c|c|c|}
\hline \multirow{2}{*}{ Operation } & \multicolumn{2}{|c|}{ Time Required (Seconds) } \\
\cline { 2 - 3 } & windows 8 & windows XP \\
\hline Capture Screenshot & 6 & 7 \\
\hline
\end{tabular}

Table 5 Time required taking and sending screenshot with JPEG compression.

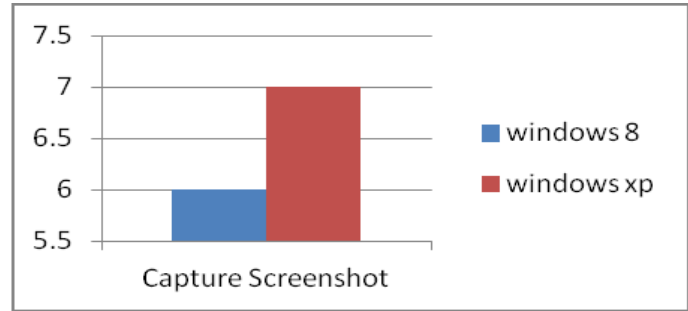

Fig 9 less time required for system with using JPEG compression

\section{Conclusion and future scope}

The system run very well on the any type of windows system which having JDK installed on it. All functions was performed correctly as tested on 20 nodes in network which includes different version of windows operating system. If JEEG algorithm was used then, it is possible to reduce time to required to capture and transfer image of screenshot over network. It helps user to see the lot of contiguous screenshot and can guess the actual state of system. In future Scope we can implement same system in Linux platform also.

\section{References}

[1] Remote Control of Mobile Devices in Android Platform , Angel Gonzalez Villan, and Josep Jorba Esteve, , IEEE 2012

[2] PocketDroid - A PC Remote Control Chaitali Navasare, Deepa Nagdev and Jai Shree, IPCSIT vol. 37 (2012)

[3] Design and Application of Remote Control System Using Mobile Phone with JNI Interface Lingyan Bi1, Weining Wang1, Haobin Zhong1,Wenxuan Liu ,International Conference on Embedded Software and Systems Symposia (ICESS2008) .

[4] Vnc Architecture Based Remote Desktop Access Through Android Mobile Phones, Archana Jadhav , International Journal of Advanced Research in Computer and Communication Engineering Vol. 1, Issue 2, April 2012.

[5] Adam, Skurski, Bartlomiej Swiercz, "VNC- based Remote Control for Symbian OS smartphones", MIXDES (Mixed Design of Integrated Circuits and Systems) 2009, June 25-27, 2009

[6] A Review of Image Compression and Comparison of its Algorithms Sachin Dhawan, IJECT Vol. 2, Issue 1 , March 2011.

[7] www.jpeg.org

[8] GPRS Based Intranet Remote Administration GIRA ,Shashi Kumar N.R. ,R Selvarani ,Pushpavathi T.P. , JRI, Volume 1 Issue 1 December 2008 